\title{
Dilema del Prisionero. Estudio de algunos aspectos del primer torneo entre programas de computadora de Axelrod
}

\author{
The Prisioner's Dilemma. A study of some \\ aspects of the first tournament between computer \\ programs by Axelrod
}

ANTONIO BENÍTEZ LÓPEZ

Universidad Complutense de Madrid

Recibido: 02/09/2016 Aceptado:16/03/2017

RESUMEN

En 1981 dio a conocer Axelrod el planteamiento y los resultados de un torneo entre programas de computadora mediante el cual intentó fijar cuál es la mejor estrategia para jugar al Dilema del Prisionero iterado. Cada programa codificó una estrategia. La estrategia ganadora, según Axelrod, fue la conocida como Tit for Tat, codificada por el programa presentado por Anatol Rapoport.

El trabajo de Axelrod es experimental. Por tanto, ha de ser posible su repetición por cualquiera. Este ensayo es un estudio de algunos aspectos de dicho experimento. En primer lugar, de los conceptos de «torneo tipo liga» y «ganador de un torneo tipo liga». En segundo lugar, de los resultados de someter a análisis la tabla 2 de Axelrod.

(C) Contrastes. Revista Internacional de Filosofía, vol. XXIII-Nº 1 (2018), pp. 7-20. ISSN: 1136-4076

Departamento de Filosofía, Universidad de Málaga, Facultad de Filosofía y Letras Campus de Teatinos, E-29071 Málaga (España) 
Quizá la conclusión principal de este estudio es que para declarar ganadora a una estrategia (programa) hay que tener en cuenta no solo el punto de vista de la cooperación (minimización del daño infligido mutuamente) sino también el punto de la vista de la confrontación (ganar a toda costa).

\title{
PALABRAS CLAVE \\ DILEMA DEL PRISIONERO, AXELROD, TORNEO COMPUTERIZADO, FILOSOFÍA EXPERIMENTAL
}

\begin{abstract}
In 1981, Axelrod announced the approach and results of a tournament between computer programs by which he tried to fix what the best strategy for playing the iterated Prisoner's Dilemma is. Each program encoded a strategy. The winning strategy, according to Axelrod, was known as Tit for Tat, encoded by the program submitted by Anatol Rapoport.

As Axelrod's work is experimental, it should be possible for anyone to repeat the experiment. This trial is a study of some aspects of that experiment. First, the concepts of «league tournament type» and «winner of a tournament league type». Third, the results of subjecting to analysis Table 2 of Axelrod.

Perhaps the main conclusion of this study is that declaring a strategy (program) as winner should take into account not only the point of view of cooperation (minimizing mutual damage) but also the point of view of the confrontation (winning at all costs).

KEYWORDS

PRISONER'S DILEMMA, AXELROD, COMPUTER TOURNAMENT, EXPERI〉MENTAL PHILOSOPHY
\end{abstract}

\section{Dilema DEL PRISIONERO}

ES USUAL PRESENTAR INTUITIVAMENTE EL LLAMADO Dilema del prisionero así: la policía arresta a dos individuos - llamemos «A» a uno, $\mathrm{y}$ «B» al otropor haber cometido juntos un crimen. La policía los mantiene en celdas separadas y ofrece a cada uno la libertad si hace una declaración inculpando al otro. La separación entre los presos tiene como consecuencia que no pueden comunicarse entre sí y tampoco saber si el otro ha declarado, y menos aún qué ha declarado.

Cada preso conoce su situación legal. La situación legal de los dos presos se entiende mejor si se considera qué pasará con ellos según que declaren contra el otro o no. Pueden pasar tres cosas:

1. Uno declara contra el otro y este no declara contra el primero. Entonces este segundo será condenado a cadena perpetua y el primero será liberado.

2. Ambos declaran contra el otro. Entonces ambos serán condenados a cárcel pero un número de años no muy grande, sea diez años.

3. Ambos se niegan a declarar. Entonces ambos serán condenados a cárcel pero un número de años reducido, sea dos años. 
Si ahora pensamos en qué pasaría si ambos presos pudieran coincidir en la misma celda y hablar, probablemente se dirían lo siguiente: «declarando uno contra el otro es posible quedar en libertad, pero si no llegamos a un trato, ambos iremos a declarar contra el otro y nos caerán diez años. Como ninguno de los dos somos estúpidos, no nos fiáremos de no declarar hasta no ver que en la declaración firmada por el otro no se nos acusa. Por tanto, no hay modo de escapar a la cárcel. Lo mejor, porque entraña la pena menor es negarse a declarar. ¿Estás de acuerdo? », dice uno.

\section{1 DiLEMA DEL PRISIONERO ITERADO}

La cosa cambia si cambiamos la escena. Seguiremos suponiendo dos actores, cada uno con sus intereses y, por ello, razonablemente egoísta. Pero lo que más cambia es que el drama no es estático sino dinámico, es decir, la situación cambia y se conservan las decisiones tomadas, al menos, en la memoria de cada uno de los actores. Sigue habiendo dos comportamientos posibles en cada situación: «cooperar» y «no cooperar», no declarar o declarar contra el otro respectivamente. Pero a cada situación producida por las decisiones de los dos actores le sigue otra un cierto número de veces. ¿Es posible actuar con una estrategia prefijada, esto es, siguiendo reglas que necesariamente dirán relación a las decisiones tomadas en el pasado? ¿Existe una estrategia ganadora?

\section{I.2 UN LENGUAJE PARA REPRESENTAR EL PROBLEMA}

- Signos: $\{1,0\}$

Leyenda:

- 1: cooperar, o no declarar

- 0: no cooperar, o declarar contra el otro

- Representación de una situación cualquiera

\begin{tabular}{c|c} 
decisión del actor $A$ & decisión del actor $B$ \\
\hline 100 & 100
\end{tabular}

Ejemplos:

- (1 1), ambos han cooperado

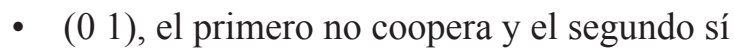


- El número de situaciones distintas es:

\begin{tabular}{c|c} 
decisión de $A$ & decisión de $B$ \\
\hline 1 & 1 \\
1 & 0 \\
0 & 1 \\
0 & 0
\end{tabular}

Es decir, el número de combinaciones, o situaciones, posibles es $2^{2}=4$.

- Es necesario advertir que cualquier situación, ya sea la única en que dos agentes toman una decisión ya sea que cada situación se inscriba en un largo curso de interacciones o secuencia de situaciones, será una, y solo una, de las determinadas por la combinatoria anteriormente reseñada.

- Puntuaciones obtenidas por cada uno de los dos actores en cada situación posible.

\begin{tabular}{|c|c|c|c|}
\multicolumn{2}{|l|}{ Situación } & \multicolumn{2}{l|}{} \\
\hline$A$ & B & Puntos $A$ & Puntos $B$ \\
\hline 0 & 1 & 5 & 0 \\
\hline 1 & 0 & 0 & 5 \\
\hline 1 & 1 & 3 & 3 \\
\hline 0 & 0 & 1 & 1 \\
\hline
\end{tabular}

Tabla 1: Criterio $D P$

- Repárese en que las puntuaciones establecidas en la tabla son arbitrarias, pero cumplen algo esencial: caso de que uno coopere y el otro no coopere, este ultimo se lleva la máxima puntuación porque quedaría en libertad y el que sí ha cooperado obtendría cero puntos porque recibiría cadena perpetua. Además las puntuaciones otorgadas tanto en el caso de la cooperación mutua como en el caso de la mutua no cooperación han de ser menores que la otorgada como puntuación máxima en el 
primer caso. Y, por último, la puntuación obtenido por no cooperar ambos ha de ser menor que la obtenida por cooperar ambos. Es decir, es posible otra tabla de puntuaciones, por ejemplo:

\begin{tabular}{|c|c|c|c|}
\multicolumn{2}{|c|}{ Situación } & \\
\hline$A$ & $B$ & Puntos $A$ & Puntos $B$ \\
\hline 0 & 1 & 10 & 0 \\
\hline 1 & 0 & 0 & 10 \\
\hline 1 & 1 & 5 & 5 \\
\hline 0 & 0 & 3 & 3 \\
\hline
\end{tabular}

He adoptado la tabla primera porque es la que más suele aparecer en la literatura sobre el Dilema del prisionero. ${ }^{1}$

Podemos considerar dos agentes que interaccionan entre sí a lo largo de bastante tiempo. El número de interacciones puede ser muy grande, pero no infinito. Simbólicamente podemos adoptar las representaciones siguientes:

- La decisión de un agente en un instante $t$ del tiempo: $D_{t}^{A}$ o $D_{t}^{B}$

- La estrategia seguida por un agente: $\mathrm{E}^{\mathrm{A}} \mathrm{O} \mathrm{E}^{\mathrm{B}}$

- Situación actual, determinada por las decisiones de los agentes: $\mathrm{S}_{\mathrm{t}}=\left(\begin{array}{ll}D_{t}^{A} & D_{t}^{B}\end{array}\right)$

- Situación precedente: $\mathrm{S}_{\mathrm{t}-1}=\left(\begin{array}{ll}D_{t-1}^{A} & D_{t-1}^{B}\end{array}\right)$

La decisión de un agente es una función cuyos argumentos son una o más situaciones precedentes y su estrategia:

- Serie de situaciones: $S_{t-1}^{t-n}$, una serie consecutiva de situaciones precedentes desde la anterior, $\mathrm{S}_{\mathrm{t}-1}$, a la más alejada en el tiempo, ${ }_{\mathrm{St}-\mathrm{n}}$

- $D_{t}^{A}=f\left(S_{t-1}^{t-n}, E^{A}\right)$

- $D_{t}^{B}=f\left(S_{t-1}^{t-n}, E^{B}\right)$

1 Axelrod 1984, p. 8. Mitchell 2009 p. 213. Binmore 2007, p. 18, propone puntuaciones alternativas. 


\section{I.3 Los trabajos de AXELRod SOBRE EL Dilema DEL PRISIONERo ItERADO}

En 1981 Axelrod dio a conocer el planteamiento y los resultados de un torneo entre estrategias mediante el cual pretendió conocer cuál es la mejor estrategia para «jugar» al Dilema del prisionero iterado. Se trató de un trabajo experimental. Cada estrategia - excepto una - fue propuesta por un experto en teoría de juegos y codificada en un programa de computadora. Fueron catorce en total más otra que Axelrod llamó «ramdon», consistente en que en cada situación «lanza una moneda» para determinar su decisión. Axelrod ideó un torneo o competición en que cada una de estas quince estrategias competía contra todas las demás y contra sí misma. El número de iteraciones fue doscientos. En cada situación, los dos contrincantes obtenían una puntuación según la tabla 1. Se suman las puntuaciones de las doscientas iteraciones de cada contrincante. El que obtenga mayor número de puntos gana la partida. Y el participante que sume el mayor número de puntos gana el torneo.

Tiene sentido que cada programa compita contra sí mismo porque algunos de ellos codificaron estrategias no deterministas. El caso extremo es el de la estrategia de jugar al azar, tirar una moneda. Es sumamente improbable que la moneda tirada al aire por el jugador $A$ y la tirada por el jugador $B$ coincidan las doscientas veces.

Axelrod dio a conocer los resultados y cierta descripción de las estrategias codificadas en dos publicaciones: «Effective Choice in the Prisoner's Dilemma» (1980) y The Evolution of Cooperation (1984).

Mi trabajo tiene como fin revisar los datos ofrecidos por Axelrod. $\mathrm{Y}$ hacer ver que son, al menos, insuficientes. En primer lugar, porque las estrategias no son descritas formalmente, de manera que cualquiera pueda volver a codificarlas en otros tantos programas de computadora, y con ello repetir el experimento de Axelrod. En segundo lugar, porque la tabla en que Axelrod sintetiza sus resultados da una información parcial, insuficiente. Y en tercer lugar, sabiendo que la preocupación de Axelrod era encontrar la mejor estrategia que fomentara la cooperación, no deja de ser cierto que aceptando su planteamiento y criterio, y repitiendo el torneo entre estrategias deterministas de cuatro bits, esto es, con estrategias deterministas en que la única situación pasada atendida para tomar la decisión es la última - como hace Tit for Tat-, la ganadora es la estrategia que no busca la cooperación sino la confrontación.

II SOBRE PUNTOS, PARTIDOS GANADOS Y TORNEOS TIPO LIGA

En el capítulo 2 de su libro (Axelrod 1984, 30) establece Axelrod que el torneo computerizado en el que participaron 14 programas y otro más cuya estrategia es jugar al azar (lanzar una moneda), se llevaría a cabo mediante un sistema de liga. Igual afirmación se encuentra en (Axelrod 1981a, 8). 
Cualquier aficionado al fútbol sabe que en un torneo tipo liga se enfrentan todos los participantes entre sí. Y que la clasificación se establece por partido ganado. En las tablas de clasificación se suelen anotar también los goles marcados y los goles recibidos. De manera que si se produjera un empate al final del torneo entre dos equipos participantes su clasificación se resolvería mediante el llamado gol average: el promedio resultante de dividir los goles a favor por los goles en contra.

Un partido puede tener un vencedor: el equipo que ha metido más goles que el contrario, o puede acabar en empate: ambos equipos han conseguido el mismo número de goles. Cuando hay un vencedor, este suma 2 puntos y el perdedor 0 puntos. Caso de empate, cada uno suma 1 punto. $^{2}$

Estas reflexiones nos han de llevar a valorar el sistema de puntuación usado por Axelrod en sus torneos.

Lo que he llamado criterio $D P$, la tabla siguiente:

\begin{tabular}{|c|c|c|c|} 
A & B & Puntos A & Puntos B \\
\hline 0 & 1 & 5 & 0 \\
\hline 1 & 0 & 0 & 5 \\
\hline 1 & 1 & 3 & 3 \\
\hline 0 & 0 & 1 & 1 \\
\hline
\end{tabular}

sirve para establecer la puntuación alcanzada por cada contrincante en una situación. Si una partida computacional ${ }^{3}$ entre dos programas tiene 200 situaciones consecutivas, la puntuación alcanzada en cada situación por cada contrincante se suma, y el ganador de la partida es el mejor de las 200 situaciones, el que más puntos sume. En términos futbolísticos: el que más goles ha marcado.

Para simplificar la discusión supongamos una partida entre el famoso Tit for Tat y un programa cuya estrategia es cooperar siempre. Y supongamos que la partida es al mejor de 4 situaciones. El resultado siempre sería el siguiente:

$$
S_{1}=\left(\begin{array}{ll}
1 & 1
\end{array}\right) \rightarrow S_{2}=\left(\begin{array}{lll}
1 & 1
\end{array}\right) \rightarrow S_{3}=\left(\begin{array}{lll}
1 & 1
\end{array}\right) \rightarrow S_{4}=\left(\begin{array}{ll}
1 & 1
\end{array}\right)
$$

Los puntos obtenidos según el criterio $D P$ en cada situación se pueden representar así:

$$
S_{1}=(33) \rightarrow S_{2}=(33) \rightarrow S_{3}=(33) \rightarrow S_{4}=(33) \quad \text { totales: [12 12] }
$$

2 Las mayorías de ligas de fútbol ahora dan 3 puntos al vencedor.

3 Es el equivalente de un partido de fútbol. 
¿Cuál ha ganado el partido? Ninguno de los dos. Han empatado. Este resultado - empate - no cambia, no puede cambiar, por más situaciones que sumemos a la partida. En efecto, si hubieran sido 200 situaciones, cada contrincante habría obtenido 600 puntos. Empate.

Supongamos, ahora, un torneo entre tres participantes: Tit for Tat, la estrategia de cooperar siempre y la estrategia de no cooperar. E igualmente por mor de la simplicidad de la argumentación, supongamos que cada partida o partido se juega a 4 situaciones.

\begin{tabular}{|c|c|c|}
\hline Prog vs. Prog & & |Totales \\
\hline TFT - COOP & $\left(\begin{array}{llllll}(1 & 1\end{array}\right)\left(\begin{array}{llll}1 & 1\end{array}\right)\left(\begin{array}{lll}1 & 1\end{array}\right)\left(\begin{array}{lll}1 & 1\end{array}\right)$ & [lll 12 12] \\
\hline TFT - NO COOP & 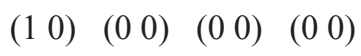 & {$\left[\begin{array}{ll}3 & 8\end{array}\right]$} \\
\hline COOP - NO COOP & $\left(\begin{array}{llll}1 & 0\end{array}\right)\left(\begin{array}{lll}1 & 0\end{array}\right)\left(\begin{array}{lll}1 & 0\end{array}\right)\left(\begin{array}{lll}1 & 0\end{array}\right)$ & {$\left[\begin{array}{ll}0 & 20\end{array}\right]$} \\
\hline
\end{tabular}

Si hubiera una segunda vuelta, los resultados serían:

\begin{tabular}{|c|c|c|}
\hline Prog vs. Prog & & |Totales \\
\hline COOP - TFT & 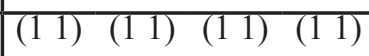 & [lll 12 12] \\
\hline NO COOP - TFT & $\left(\begin{array}{llll}0 & 1\end{array}\right)\left(\begin{array}{lll}0 & 0\end{array}\right)\left(\begin{array}{lll}0 & 0\end{array}\right)\left(\begin{array}{lll}0 & 0\end{array}\right)$ & [[ 8 r 3] \\
\hline NO COOP - CoOP & $\left(\begin{array}{lllll}0 & 1\end{array}\right)\left(\begin{array}{llll}0 & 1\end{array}\right)\left(\begin{array}{lll}0 & 1\end{array}\right)\left(\begin{array}{lll}0 & 1\end{array}\right)$ & |[ $\left[\begin{array}{ll}2 & 0\end{array}\right]$ \\
\hline
\end{tabular}

Bien, pero ¿quién ganó este campeonato? El primer partido o partida terminó en empate. Los dos siguientes los ganó el no cooperante. Por tanto, la tabla de clasificación hay que establecerla así:

\begin{tabular}{|l|l|c|c|c|c|c|l|}
\hline orden & programa & jugados & ganados & empatados & perdidos & puntos Liga & puntos DP \\
\hline 1 & NO COOP & 2 & 2 & 0 & 0 & $2 \times 2=4$ & $8+20=28$ \\
\hline 2 & TFT & 2 & 0 & 1 & 1 & $1 \times 1=1$ & $12+3=15$ \\
\hline 3 & COOP & 2 & 0 & 1 & 1 & $1 \times 1=1$ & $12+0=12$ \\
\hline
\end{tabular}

En esta tabla se ha supuesto que una partida ganada vale 2 puntos, una partida empatada vale 1 punto y 0 puntos la perdida. El equivalente a goles a favor en el fútbol es aquí el total de puntos según el criterio $D P$.

El ganador, por tanto, es la estrategia no cooperante porque gana 2 partidas. Tit for Tat y la estrategia cooperante obtienen los mismos puntos. 
La ordenación final es debida a que Tit for Tat tiene más puntos DP que la estrategia cooperante.

Ahora se puede concluir:

1. El ganador de una partida es aquel que más puntos $D P$ obtiene. A éste se le darán 2 puntos como vencedor de la partida, y al perdedor 0 puntos. $\mathrm{Si}$ ambos hubieran obtenido igual número de puntos $D P$, ambos recibirían 1 punto.

\section{II.1 GANADOR SEGÚN AXELROD}

Si se estudia la tabla $2^{4}$ : Tournament Scores, se advierte de inmediato que Axelrod declara vencedor al programa que más puntos $D P$ ha obtenido, y no aquel que más partidas ha ganado.

Aparentemente es una extraña decisión. Es semejante a decidir el vencedor de la final de la NBA por la suma de puntos marcados y no por la suma de partidos ganados. En la final de 2016 los Cleveland Cavaliers sumaron 670 puntos, y los Golden State Warriors 732. Pero los Cleveland Cavaliers ganaron 4 partidos frente a 3 los Golden State Warriors. El ganador de la final fue el que más partidos ganó: Cleveland Cavaliers.

Mi conclusión es:

1. Hay que encontrar una explicación para el criterio seguido por Axelrod para decidir el vencedor de un torneo. 


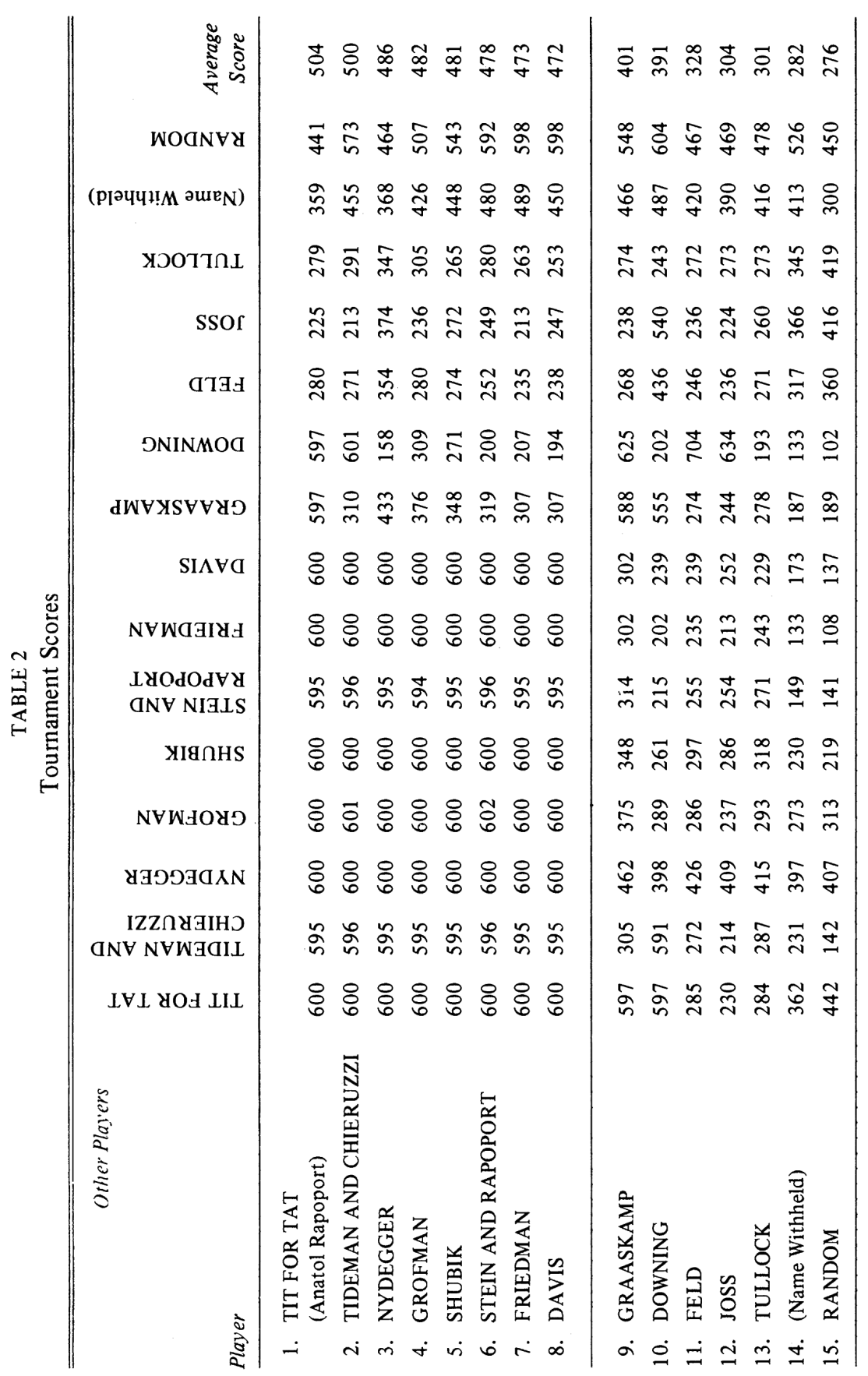




\section{II.2 ANÁLISIS DEL TORNEO SEGÚN LA TABLA 2}

Si se añade a la tabla de Axelrod los resultados de partidas ganadas, empatadas o perdidas, se tiene la siguiente tabla:

\begin{tabular}{|c|c|c|c|c|c|c|c|c|c|}
\hline 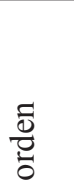 & 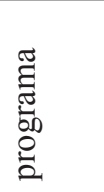 & 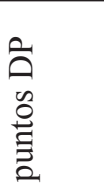 & 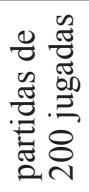 & 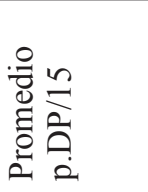 & 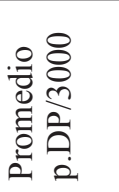 & 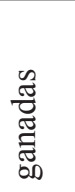 & 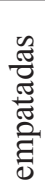 & 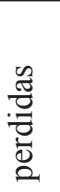 & 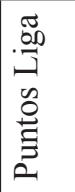 \\
\hline 1 & $\mathrm{tft}$ & 7568 & 15 & 504,533 & 2,5226 & 0 & 8 & 7 & 8 \\
\hline 2 & tid-ch & 7507 & 15 & 500,4666 & 2,5023 & 11 & 2 & 2 & 24 \\
\hline 3 & nyd & 7288 & 15 & 485,8666 & 2,4293 & 1 & 6 & 8 & 8 \\
\hline 4 & grof & 7228 & 15 & 481,8666 & 2,4093 & 5 & 6 & 4 & 16 \\
\hline 5 & shu & 7211 & 15 & 480,7333 & 2,4036 & 3 & 7 & 5 & 13 \\
\hline 6 & str & 7166 & 15 & 477,7333 & 2,3886 & 10 & 2 & 3 & 22 \\
\hline 7 & fried & 7102 & 15 & 473,4666 & 2,3673 & 5 & 8 & 2 & 18 \\
\hline 8 & dav & 7077 & 15 & 471,8 & 2,359 & 4 & 6 & 5 & 14 \\
\hline 9 & graas & 6012 & 15 & 400,8 & 2,004 & 4 & 3 & 8 & 11 \\
\hline 10 & down & 5859 & 15 & 390,6 & 1,953 & 6 & 2 & 7 & 14 \\
\hline 11 & feld & 4914 & 15 & 327,6 & 1,638 & 12 & 3 & 0 & 27 \\
\hline 12 & joss & 4565 & 15 & 304,3333 & 1,5216 & 12 & 3 & 0 & 27 \\
\hline 13 & tull & 4509 & 15 & 300,6 & 1,503 & 6 & 1 & 8 & 13 \\
\hline 14 & nw & 4235 & 15 & 282,3333 & 1,4116 & 3 & 1 & 11 & 7 \\
\hline 15 & ram & 4145 & 15 & 276,3333 & 1,3816 & 1 & 1 & 13 & 3 \\
\hline
\end{tabular}

He de aclarar, inmediatamente, cómo he determinado las puntuaciones obtenidas por cada participante en un enfrentamiento o partida. En el enfrentamiento entre Tit for Tat y Tideman and Chieruzzi he considerado que Tit for Tat obtuvo 595 puntos DP: es la cifra existente en la intersección entre la fila 1 y la columna de nombre «Tideman and Chieruzzi» en la tabla 2. La puntuación de Tideman and Chieruzzi la he obtenido de la intersección entre la fila 2 y la columna de nombre «Tit for Tat»: 600 puntos DP. Para los enfrentamientos de cada participante consigo mismo he buscado la intersección entre la fila y la columna de su nombre. 
Es posible que estos no fueran los resultados obtenidos por Axelrod para los programas que juegan en posición 2. ¿Por qué dudo? Por el caso del programa random. En efecto, es muy dudoso que la puntuación de random en posición 1 y random en posición 2 coincidieran, pero el método señalado en el párrafo anterior obliga a creerlo.

Pero Axelrod no los da en ningún sitio. A mi juicio, Axelrod debería haber dado las puntuaciones alcanzadas por cada uno de los dos programas en cada enfrentamiento. Pero el caso es que no disponemos de esa información.

Como puede apreciarse la diferencia porcentual entre Titfor Tat y Tideman and Chieruzzi es de 2 centésimas: 2,5226 frente a 2,5023. La diferencia en puntos DP absolutos es de 61. Por estas razones Axelrod proclamó ganador del torneo a TIT FOR TAT.

No obstante, llama la atención el mal desempeño de TIT FOR TAT desde el punto de vista de una competición pura tipo liga: no gana ni un solo partido, empata en 8 ocasiones y pierde en 7 .

Tideman and Chieruzzi se desempeña bien: gana 11 encuentros, empata en 2 ocasiones y pierde en 2. Pierde con Feld y Joss por 1 punto. ¿No hay que pensar que la estrategia de Tideman and Chieruzzi es más equilibrada que la de Tit for Tat? , puesto que consigue una puntuación DP muy cercana a la de esta última y, sin embargo, tiene un desempeño muchísimo mejor que Tit for Tat. O dicho de otra manera, cuando se opone a estrategias que buscan el enfrentamiento, la confrontación, es capaz de ganar. Y cuando se enfrenta a estrategias que buscan la cooperación también es capaz de ganar.

\section{3 EL PUNTO DE VISTA DE LA COOPERACIÓN}

La tabla anterior deja ver claramente cuál es el punto de vista de la cooperación.

Cuando dos agentes compiten en el marco del Dilema del prisionero, caben dos perspectivas desde las que analizar los resultados de la confrontación.

El primer punto de vista es el de la confrontación. Los dos prisioneros compiten con la intención de liberarse a sí mismo. Por tanto, lo importante es quien gana el duelo, la competición. ¿Quién gana el torneo, según las tablas anteriores, desde el punto de vista de la confrontación? Las estrategias de Feld y Joss, pues no perdieron ningún enfrentamiento. Empataron 3 veces y ganaron 12 confrontaciones cada uno.

El segundo punto de vista es el de la cooperación. Los dos prisioneros compiten con la intención de minimizar el daño que se pueden infligir mutuamente. Por tanto, lo importante es obtener una puntuación lo más alta que se pueda. Para ello, los prisioneros han de elegir no declarar contra el otro, callar. Lo importante, desde este punto de vista, es obtener una puntuación alta, y carece de interés ganar la confrontación. Las estrategias de Tit for Tat 
y Tideman and Chieruzzi son las dos mejores tanto en puntos DP como en porcentaje.

Creo que hay tres conclusiones que añadir a las ya estudiadas:

1. Hay dos posibles puntos de vista en el análisis de torneos en se que enfrentan estrategias para dirimir el Dilema del prisionero: confrontación y cooperación.

2. Si se miden los resultados de un torneo por las partidas ganadas, entonces se adopta el punto de vista de la confrontación.

3. Si se miden los resultados de un torneo por el promedio de puntos DP obtenidos, entonces se adopta el punto de vista de la cooperación. Este es el punto de vista que Axelrod adoptó.

\section{CONCLUSIONES}

Intentaré ser muy escueto al presentar mis conclusiones.

1. El torneo computerizado entre programas que codifican estrategias para jugar al Dilema del Prisionero iterado es un trabajo experimental. Por ello, ha de ser posible su repetición por cualquiera y en cualquier momento.

i. Para repetirlo, es necesario contar con una definición formal de cada una de las estrategias codificadas en programas. Por ejemplo, la estrategia conocida como Tit for Tat puede ser definida formalmente así:

$\mathrm{R} 1: \sin n=1$, decisión $=1$

[para $n=$ número de iteración]

R2: else, decisión $=($ last (first serie-iteraciones $))$

[para serie-iteraciones $\left.\left.=\left(\begin{array}{lll}1 & 1\end{array}\right)\left(\begin{array}{lll}0 & 1\end{array}\right)\left(\begin{array}{ll}1 & 0\end{array}\right) \ldots\right)\right]$

Es decir, mediante un sistema de reglas de producción.

ii. Las descripciones contenidas en el Apéndice «The Decision Rules» son insuficientes y no hacen posible ni una codificación ni una definición formal de las distintas estrategias.

iii. Hay que dar la información de los puntos DP obtenidos por cada programa interviniente en un partido.

2. Si el torneo tenía el objetivo de estudiar cuándo y cómo una persona, país o institución, debería cooperar con otra persona, país o institución, y cuándo conviene no cooperar, parece relevante tener en cuenta tanto la puntuación DP como la puntuación Liga. Si se hace así, es dudoso que el ganador del torneo fuera Tit for Tat. 


\section{REFERENCIAS BIBLIOGRÁFICAS}

AXELROD, R. 1980: «Effective Choice in the Prisoner's Dilemma». The Journal of Conflict Resolution, Vol. 24, No. 1, pp. 3-25.

AXELROD, R. 1980: «More Effective Choice in the Prisoner's Dilemma». The Journal of Conflict Resolution, Vol. 24, No. 3 , pp. 379-403.

AXELROD, R. 1984: The Evolution of Cooperation. N. York: Basic Books Inc. Publisher.

AXELROD, R. 1997: The complexity of cooperation : agent-based models of competition and collaboration. Princeton (N. J.): Princeton University Press.

BINMORE, K. 2007: Game Theory. A Very Short Introduction. Oxford: Oxford U. Press.

HO, T-H. 1996: «Finite automata play repeated prisoner's dilemma with information processing costs». Journal of Economic Dynamics and Control 20, pp. 173-207.

HOFSTADTER, D. R. 1983: "Computer tournaments of the Prisoner's Dilemma suggest how cooperation evolves». Scientific American, Volume 248, Issue 5, pp. 16-26.

MITCHELL, M. 2009: Complexity. A Guided Tour. N. York: Oxford University Press.

RUBINSTEIN, A. 1986: «Finite Automata Play the Repeated Prisoner's Dilemma». Journal of Economic Theory 39, pp. 83-96.

DE SANTIAGO, R. y GARCÍA, J.A. 1994: «¿Es posible ganar a Toma-y-Daca? ». Anales de Estudios Económicos y Empresariales, Vol. 9, pp. 159-184.

SIMON, H. 1996: The Science of the Artificial. Cambridge (MA): The MIT Press.

Antonio Benítez es profesor jubilado del Departamento de Lógica y Filosofía de la Ciencia de la Universidad Complutense de Madrid.

Líneas de Investigación:

Inteligencia Artificial, Estudios de los sistemas complejos, Filosofía la mente, Lógica

Publicaciones recientes:

(2016): «Self-Reproduction of an Artificial Animal. Second Model», Journal of Communication and Computer, 8 (June). ISSN 1548-7709).

(2015): Lógicas no clásicas. Una introducción. Madrid: Escolar y Mayo editores, 2015. ISBN: 978-84-16020-25-6.

Correo electrónico: abenitez@ucm.es 